\title{
Comparison of single and two-tunnel techniques during open treatment of acromioclavicular joint disruption
}

Zhiyong Hou ${ }^{\text {* }}$, Jove Graham², Yingze Zhang ${ }^{1}$, Kent Strohecker², Daniel Feldmann², Thomas R Bowen², Wei Chen ${ }^{1}$ and Wade Smith $^{3}$

\begin{abstract}
Background: Coracoclavicular (CC) ligament reconstruction with semitendinosus tendon (ST) grafts has become more popular and has achieved relatively good results; however optimal reconstruction technique, single-tunnel or two-tunnel, still remains controversial. This paper is to compare the clinical and radiographic data of allogenous ST grafting with single- or two-tunnel reconstruction techniques of the AC joint.

Methods: The outcomes of 21 consecutive patients who underwent anatomical reduction and ST grafting for AC joint separation were reviewed retrospectively. Patients were divided into two groups: single-tunnel group (11) and two-tunnel group (10). All patients were evaluated clinically and radiographically using a modified UCLA rating scale.

Results: The majority of separations (18 of 21) were Rockwood type V, with one each in type III, IV and VI categories. The overall mean follow-up time was 16 months, and at the time of the latest follow-up, the overall mean UCLA rating score was 14.1 (range 8-20).

The percentage of good-to-excellent outcomes was significantly higher for patients with the two-tunnel technique than for those with the one-tunnel technique ( $70 \%$ vs. $18 \%$, respectively, $p=0.03)$. Within the single-tunnel group, there was no statistically significant difference in percentage of good-to-excellent outcomes between patients with vs. without tightrope augmentation ( $17 \%$ vs $20 \%, p>0.99$ ). Similarly, within the two-tunnel group, there was no significant difference in the percentage of good-to-excellent outcomes between the graft only and augment groups (67\% vs. $75 \%, p>0.99)$.

Conclusion: Anatomical reduction of the AC joint and reconstruction CC ligaments are crucial for optimal joint stability and function. Two-tunnel CC reconstruction with an allogenous ST graft provides superior significantly better radiographic and clinical results compared to the single-tunnel reconstruction technique.
\end{abstract}

Keywords: Acromioclavicular joint, Single-tunnel, Two-tunnel, Reconstruction, Augmentation

\section{Background}

Acromioclavicular $(\mathrm{AC})$ joint injuries are among the most commonly occurring problems in the young and active patient population. Higher-grade AC joint injuries (Rockwood types III through VI) represent failure of the coracoclavicular (CC) ligament complex, which is formed by the conoid and trapezoid ligaments. This complex has been termed the primary suspensory structure of the

\footnotetext{
* Correspondence: drzyhou@gmail.com

'Department of Orthopaedic Surgery, Third Hospital of Hebei Medical University, Shijiazhuang, Hebei 050051, China

Full list of author information is available at the end of the article
}

upper limb [1,2]. In the literature, the incidence of traumatic AC joint separation varies from 3 to 4 per 100,000 people with $25-52 \%$ of these occurring during sporting activities, and they are also one of the most common shoulder injuries seen in orthopaedic traumatology [2-5]. For certain Rockwood type III AC joint separations and all type IV, V, and VI injuries, surgical treatment has been recommended to prevent disabling pain, weakness, and deformity [6-8]. Although more than 60 surgical techniques have been reported, the frequency of failure to maintain reduction after surgical treatment remains high $[9,10]$. 
Recently, CC ligament reconstruction with tendon grafts has become more popular and has achieved relatively good results $[11,12]$. Biomechanical studies focusing on an anatomic reconstruction of the $\mathrm{CC}$ ligament complex using tendon grafts have reported promising potential for this technique [13-15]. Semitendinosus tendon (ST) grafting and anatomic reconstruction can be imitated, providing stability to the clavicle that is very close to that provided by the intact ligaments [13]. However, optimal reconstruction technique, single-tunnel or two-tunnel, still remains controversial. Anatomical two-tunnel reconstruction with tendon grafts or synthetic materials seems appealing because it has been shown by biomechanical studies to restore the original two ligaments (the conoid and trapezoid) and to produce an ultimate failure load that is equivalent to that of native CC ligaments [13-15]. However, it is technically difficult and theoretically increases the risk of fracture [16].

The purpose of this retrospective study was to analyze the clinical and radiographic data of allogenous ST tendon grafting with single- or two-tunnel reconstruction techniques of the $\mathrm{CC}$ ligaments. We hypothesize that anatomic reconstruction of the $\mathrm{AC}$ joint disruption using two-tunnel reconstruction technique results in a satisfying clinical function and provides stable fixation.

\section{Methods}

Between June 2003 and January 2009, twenty-three patients underwent open operation for AC joint reconstruction with ST allograft at our institution. In the earlier study period before 2007, we mostly used single-tunnel technique, and after 2007 mostly the two-tunnel technique. For analysis we divided patients into two groups: singletunnel group and two-tunnel group. Patient data were collected retrospectively, including gender, age at the time of surgery, injury mechanism, classification according to Rockwood, and surgical technique. Patients with at least 12 months of clinical follow-up were included in this study. Patients were excluded if they had a previous shoulder injury, arthritis, or an associated neurological deficit on the side of injury.

The procedure was performed with the patient in the beach chair position under general anesthesia in combination with an interscalene block. An anterior deltopectoral approach was utilized with saber incision, The AC joint, the lateral end of the clavicle, and the coracoid process were exposed. Subperiosteal detachment of the deltotrapezial fascia from the clavicle was performed. The distal end of the clavicle was resected 8 to $10 \mathrm{~mm}$ using an oscillating saw. For the single-tunnel technique, a $6-\mathrm{mm}$ drill hole was made about $1.5-2 \mathrm{~cm}$ medial to the remaining end of the clavicle superior to inferior in a 300 posterior to anterior angle. A ST allograft was prepared by placing a whipstitch (Arthrex \#2 Fiberwire suture, Naples, FL, USA) on either end. After reducing the distal clavicle down to the acromion anatomically, the ST graft was introduced around the base of the coracoid and then both ends of the graft up through the clavicle hole. The graft was then mechanically tensioned and a $5.5 \mathrm{~mm}$ Bio-tenodesis screw was placed down through the center of the ST graft fixing it to the clavicle. The free ends of the graft were then passed underneath the clavicle and tied to themselves for additional fixation (Figure 1). If using a tightrope augment (Arthrex Fiberwire No. 5, Naples, FL, USA), a guide was used to place a pin from a point medial to the lateral tunnel, to the base of the coracoid. A $4.5 \mathrm{~mm}$ reamer was then used to create a tunnel through the clavicle and coracoid. The tight rope device was placed through the clavicular and then coracoid tunnel and endobutton secured against inferior cortex of coracoid. The tight-rope was then tied after fixation of the graft. Later in the series, a single clavicular tunnel was utilized for both the graft and tight-rope. The graft was placed around the coracoid and through the clavicular tunnel and tightrope device (Figure 2).

For the two-tunnel technique, the same delto-pectoral approach was used. Two holes were drilled in the clavicle to reconstruct each of the two CC ligaments, trapezoid and conoid ligaments. The lateral tunnel is created as in the single-tunnel technique. The medial tunnel is located $4.5 \mathrm{~cm}$ medial to the AC joint. A $5.5 \mathrm{~mm}$ tunnel is reamed like the medial tunnel. A single ST graft was prepared and looped under the coracoid. The lateral free end was brought up through the lateral tunnel, and the medial free end through the medial tunnel. The AC joint is reduced, and the grafts fixed into the tunnels with $5 \mathrm{~mm}$ biotenodesis screws and the graft tied to itself (Figure 3). If using tightrope augment, a guide pin is placed between the two graft tunnels, from midline, through the clavicle and base of coracoid. A $4.5 \mathrm{~mm}$ tunnel is reamed over the guide wire and the Tight-rope device placed through the clavicle and coracoid and secured to the inferior cortex of the

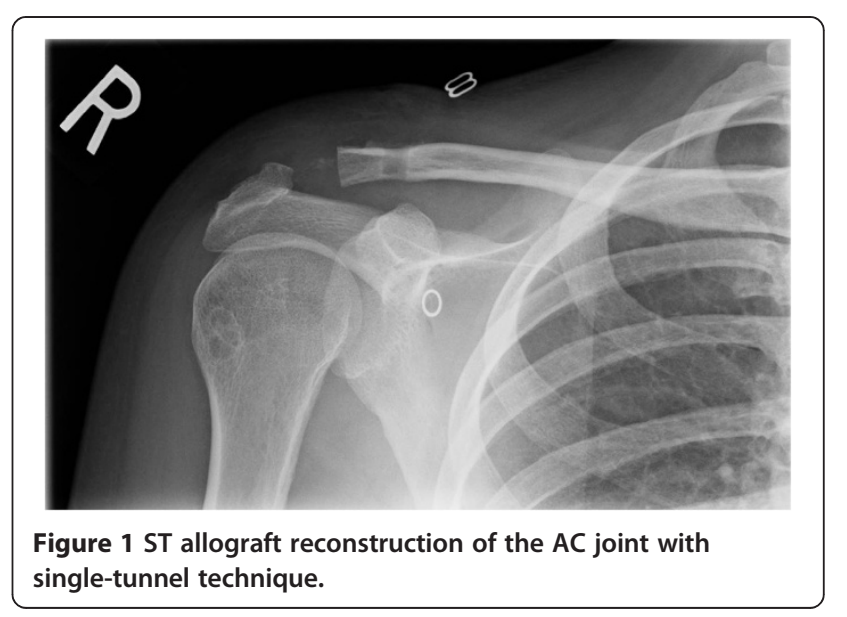




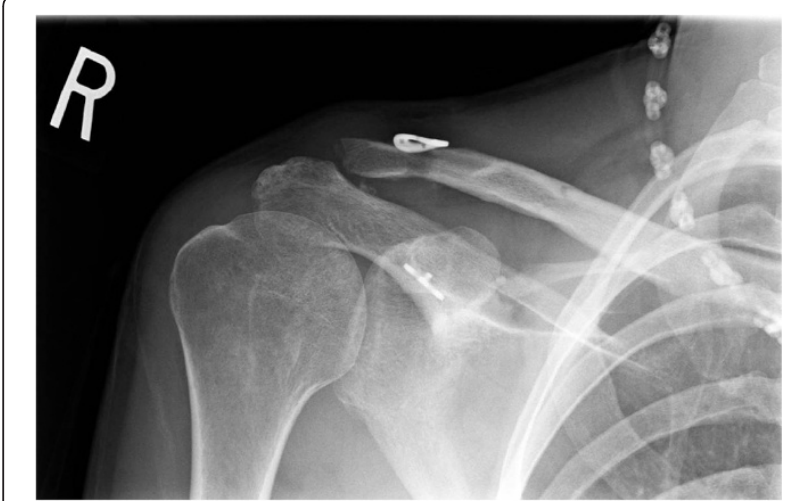

Figure 2 ST Allograft with tightrope augment reconstruction of the CC joint with single-tunnel technique.

coracoid. The device is tightened and tied after graft fixation (Figure 4). After reconstruction, attention was directed to repair of the deltotrapezial interval. This was performed in a pants-over-vest fashion using \#1 or \#2 non-absorbable sutures in an interrupted fashion. A layered closure was then performed. A drain was not utilized.

All patients were placed in a sling immobilizer post-op for 4 to 6 weeks. Gentle pendulums and Codman's were begun post-op day 1 . At 4 weeks therapy was begun with passive motion and cuff isometrics. Resistive program started at 8 weeks. Patients were generally allowed to return to manual work and athletics at 4 to 6 months depending on level of rehabilitation. Contact sports not prior to six months. All patients were evaluated clinically and radiographically using a modified UCLA rating scale [5,17], which reflects three parts: maintenance of reduction, objective evaluation of the patient's function, and complications secondary to operation. In the radiological evaluation, the roentgenographic rating was determined by the degree of displacement of the AC joint, which

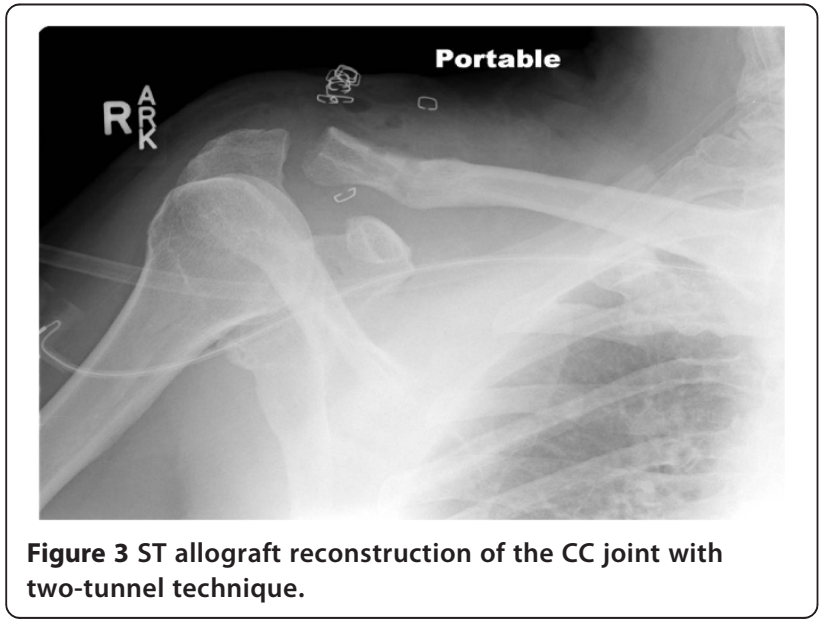

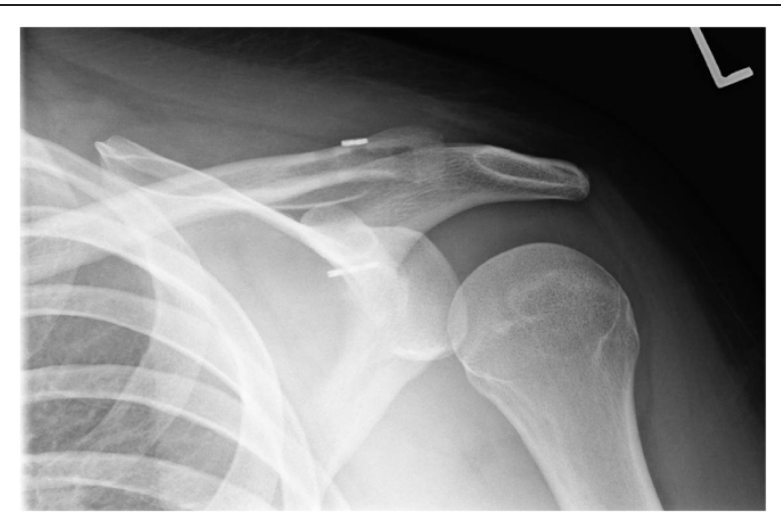

Figure 4 ST Allograft with tightrope augment reconstruction of the CC joint with two-tunnel technique.

was evaluated by measuring the relation between the acromion and the clavicle on the anteroposterior view for vertical displacement (reduced $=4$ points, subluxed $=2$ points, dislocated $=0$ points). In the physical evaluation, range of motion (ROM), pain, weakness, and complications were recorded. Finally, patients were asked their overall satisfaction with the postoperative result, with 0 points for dissatisfaction or unsure and 2 points for satisfaction.

Table 1 shows the relative weight given to each category of the rating scale and describes the criteria by which a patient was assigned an overall final result of excellent, good, fair, or poor.

Percentages of good-to-excellent outcomes and maintenance of reduction (reduced or subluxed) were compared between the two reconstruction procedures (single vs. two-tunnel), and between augmentation techniques (with vs. without tightrope). Because of the relatively small sample sizes, Fisher's exact test was used in place of chi-square testing at a significance level of $\mathrm{p}<0.05$. All analysis was performed using SAS statistical software (SAS 9.2, Cary, NC). Waiver of patient consent was granted by Institutional Review Board of Geisinger Medical Center for retrospective chart review.

\section{Results}

From the initial 23 patients who were surgically treated, two patients were lost to follow up and were excluded. Table 2 summarizes the demographics and injury characteristics of the 21 patients remaining in the study. The majority of fractures (18 of 21) were Rockwood type $\mathrm{V}$, with one fracture each in type III, IV and VI categories. Most of those patients had received primary unsuccessful conservative care and switched to operative management, and one patient underwent a failed Weaver-Dunn procedure.

The overall mean follow-up time was 16 months, and at the time of the latest follow-up, the overall mean UCLA rating score was 14.1 (range 8-20). Eleven (52\%) patients 
Table 1 The modification of the UCLA rating scale ${ }^{8.17}$

\begin{tabular}{|c|c|}
\hline Category & Points \\
\hline \multicolumn{2}{|l|}{ Maintenance of reduction } \\
\hline Reduced & 4 \\
\hline Subluxion & 2 \\
\hline Dislocation & 0 \\
\hline \multicolumn{2}{|l|}{ Range of motion } \\
\hline Full & 2 \\
\hline Improved from preoperative & 1 \\
\hline No change from preoperative & 0 \\
\hline \multicolumn{2}{|l|}{ Strength } \\
\hline Normal & 2 \\
\hline Improved from preoperative & 1 \\
\hline Unimproved from preoperative & 0 \\
\hline \multicolumn{2}{|l|}{ Pain } \\
\hline None & 4 \\
\hline With strenuous activity & 3 \\
\hline With moderate activity & 2 \\
\hline With mild activity & 1 \\
\hline All the time & 0 \\
\hline \multicolumn{2}{|l|}{ Weakness } \\
\hline None & 2 \\
\hline With strenuous activity & 1 \\
\hline All the time & 0 \\
\hline \multicolumn{2}{|l|}{ Change in occupation } \\
\hline Same or more strenuous & 2 \\
\hline Less strenuous & 0 \\
\hline \multicolumn{2}{|l|}{ Complication } \\
\hline None & 2 \\
\hline Minor/resolved & 1 \\
\hline Major/affected outcome & 0 \\
\hline \multicolumn{2}{|l|}{ Patient satisfaction } \\
\hline Yes & 2 \\
\hline No or unsure & 0 \\
\hline
\end{tabular}

Results: excellent, 18-20; good, 15-17; fair, 12-14; poor, $\leq 11$.

rated the outcome as good to excellent, 3 (14\%) rated it as fair, and $7(33 \%)$ rated it as poor. Three of 21 patients underwent additional revision surgery for the failed CC ligament repair or reconstruction.

Of the 21 patients, eleven patients underwent allogenous ST grafting with single-tunnel reconstruction technique, and 6 of these received tightrope augmentation. Ten patients underwent allogenous ST grafting with two-tunnel reconstruction technique: four of these received one ST graft plus one tightrope graft ("ST-tightrope"), while the other six received two ST grafts (“ST-ST").
Table 2 Demographic and injury characteristics, by single-tunnel and two-tunnel group

\begin{tabular}{lcc}
\hline Parameter & Single-tunnel (11) & Two-tunnel (10) \\
\hline Gender & 6 & 9 \\
Male & 5 & 1 \\
Female & $37(20-55)$ & $42(20-63)$ \\
Mean age (range), years & & \\
Side of Fracture & 5 & 8 \\
Right & 6 & 2 \\
Left & & 6 \\
Mechanism of Injury & 6 & 2 \\
Sporting & 4 & 2 \\
Traffic accident & 1 & \\
Fall & & 0 \\
Rockwood Classification & 1 & 1 \\
C3 & 0 & 8 \\
C4 & 10 & 1 \\
C5 & 0 & $(12-40)$ \\
C6 & $16(12-38)$ & \\
Mean length of follow up & & \\
(range), months & & \\
\hline
\end{tabular}

Table 3 summarizes the UCLA rating scale scores at last follow-up for the two groups (single- and two-tunnel), subdivided by augmentation type. The percentage of good-to-excellent outcomes was significantly higher for patients with the two-tunnel technique than for those with the one-tunnel technique ( $70 \%$ vs. $18 \%$, respectively, $\mathrm{p}=0.03)$. Within the single-tunnel group, there was no statistically significant difference in percentage of goodto-excellent outcomes between patients with vs. without tightrope augmentation ( $17 \%$ vs $20 \%$, p > 0.99). Similarly, within the two-tunnel group, there was no significant difference in the percentage of good-to-excellent outcomes between ST-tightrope and ST-ST patients (75\% vs. $67 \%, \mathrm{p}>0.99)$.

We noted that complications were observed in three of the 21 patients: two patients in the two-tunnel group had infection, and one patient in the single-tunnel group had a coracoid fracture. Calcification of the CC ligament occurred in one case, but it did not appear to cause symptoms, and was therefore not considered a complication. No patient had neurovascular or post-traumatic arthritis of the injured AC joint.

\section{Discussion}

Our data demonstrated that allogenous ST grafting with two-tunnel reconstruction technique of the AC joint yielded excellent or good clinical outcomes more frequently compared to single-tunnel reconstruction technique. These results also suggest that the materials used 
Table 3 Number of patients receiving single-tunnel vs. two-tunnel techniques, subdivided by augmentation type, with clinical outcome results based on modification of the UCLA rating scale

\begin{tabular}{|c|c|c|c|c|}
\hline \multirow[t]{2}{*}{ UCLA rating scale } & \multicolumn{2}{|c|}{ Single-tunnel $(n=11)$} & \multicolumn{2}{|c|}{ Two-tunnel $(n=10)^{*}$} \\
\hline & With augment & Without augment $^{\wedge}$ & ST-tightrope & ST-ST \\
\hline Excellent & 1 & 1 & 1 & 2 \\
\hline Good & 0 & 0 & 2 & 2 \\
\hline Fair & 2 & 1 & 1 & 1 \\
\hline Poor & 3 & 3 & 0 & 1 \\
\hline Total & 6 & 5 & 4 & 6 \\
\hline $\mathrm{N}(\%)$ with excellent or good & $1(17 \%)$ & $1(20 \%)$ & $3(75 \%)$ & $4(67 \%)$ \\
\hline
\end{tabular}

*Two-tunnel group had significantly higher percentage of good-to-excellent outcomes than single-tunnel group, $p=0.03$.

${ }^{\wedge}$ No significant difference between with vs. without augmentation for single-tunnel group, $\mathrm{p}>0.99$.

${ }^{\#}$ No significant difference between ST-tightrope vs. ST-ST for two-tunnel group, $p>0.99$.

for augmentation in the two-tunnel reconstruction technique do not impact the clinical result. In this technique, one ST allograft combined with one tightrope graft construction can provide similar outcomes to using ST allograft in both tunnels. We also saw no significant differences between patients with and without tightrope augment in the single-tunnel technique group.

Based on well established anatomical ligament reconstruction in the knee injury, reconstructing the $\mathrm{CC}$ ligament using tendon graft for $\mathrm{AC}$ joint injury has become more popular because the construct is more physiologic, does not require implant removal and preserves the CA ligament $[18,19]$. ST tendon grafts are most common used for this procedure, which can be either autografts or allografts, and have achieved relatively good results [11-13,20,21]. The harvesting of an autogenous tendon may not result in long-term functional impairment but may still cause some morbidity associated with the donor site, and also create a second operative site during $\mathrm{AC}$ joint surgery [22]. Nicholas et al. [12] achieved excellent outcomes after fresh-frozen ST allograft reconstruction of the CC ligament; patients reported significant pain relief, return of normal strength and function, negligible loss of motion, and no loss of reduction on postoperative radiographs. Based on this information, the substitution of allograft material has become a routine procedure in our institution. The current surgical technique for the CC ligament reconstruction can be graft tendon passed though the clavicle with single tunnel or two tunnels technique $[16,23]$, looped around the base of the coracoids [24], passed through a transosseous tunnel in the coracoids [25], or fixed to the base of coracoid using an anchor technique [6]. The CC ligament is stabilized by 2 sets of ligamentous structures: the conoid and trapezoid. Single-tunnel or two-tunnel reconstruction still remains controversial. Mazzocca et al. considered that each CC ligament has a separate function, and so each must be considered in reconstructive procedures [26]. Anatomical two-tunnel reconstruction with tendon grafts has yielded good results because it restores the original 2 ligaments and produces an ultimate strength that is equivalent to that of native CC ligaments $[14,15,23]$. However, two-tunnel techniques are technically difficult, with increased risk of fracture, and sometimes are not possible in patients with a small clavicle $[13,16]$. This technique should be performed by an experienced arthroscopist [23]. Yoo et al. [16] reported that single-tunnel reconstruction has some advantages over two-tunnel techniques. They reconstructed CC ligaments in 21 patients using a single-tunnel ST autograft and achieved superior clinical result. 17 (81\%) of the 21 patients maintained complete reduction, and only 1 patient (reportedly a manual laborer) had complete reduction loss. In our cohort, there was a statistically significant difference in percentages of good-to-excellent UCLA scores between the single-tunnel and two-tunnel groups. The two-tunnel group had better scores, with the caveat that we observed two cases of infection in the two-tunnel group which may be related to the greater length and complexity of this procedure as compared to the singletunnel technique.

Anatomical two-tunnel reconstruction with ST tendon grafts or synthetic materials provided similar results. The tightrope system, consisting of one round clavicle titanium button and one long coracoid titanium button connected by non-absorbable sutures (No. 5 Ethibond suture), has been initially utilized for repair of acute syndesmosis disruptions. The application has been extended and previously described for AC joint dislocations [27,28]. It can be used as a single graft device or an augment for the other tendon graft construction. Two-tunnel reconstruction technique has been shown by biomechanical studies to restore the strength of the original two ligaments (the conoid and trapezoid) and result in significantly higher stability in the superoinferior as well as the anteroposterior plane when compared with the native CC ligaments [11,14,15,29]. Grafting materials for the two-tunnel technique use are variable, and may include two tendon grafts, two tightrope grafts, or one tendon with one tightrope 
grafts. Salzmann et al. [23] reported on 23 consecutive patients with the acute $\mathrm{AC}$ joint disruption who underwent two-tunnel anatomical reconstruction of $\mathrm{CC}$ ligaments using two flip-button tightropes. This procedure yielded satisfactory clinical function and provided a stable fixation at intermediate-term follow-up. In our two-tunnel group, most patients had good-to-excellent UCLA scores at last followup, and this result did not vary between the cases treated with one ST graft and one tightrope graft versus those treated with two ST grafts.

Augmentation has been shown to be beneficial during $\mathrm{CC}$ ligament reconstructions by biomechanical studies $[30,31]$. An effective augmentation must have biomechanical properties enabling it to shield the repair or reconstruction from excessive tensile force, ideally allowing early rehabilitation. It seems desirable for an augmentation to possess strength and stiffness similar to those of the intact CC ligament complex, thus protecting against physiologic loads while allowing for physiologic motion between the clavicle and coracoid. Tienen et al. [32] had good results with using an open modified Weaver-Dunn technique and $\mathrm{AC}$ joint augmentation with absorbable, braided suture in 21 paptients. The tightrope augmentation was initially described for acute AC joint dislocation and represented an excellent biological augmentation technique by Hernegger [27]. Scheibel et al. [33] also reported using a gracilis tendon reconstruction augmented with a tightrope achieved good and excellent results and maintained good reduction for acute AC joint dislocations with one year follow up. Recently, Yoo et al. [16] also reported a superior result by using the tightrope augment technique to protect the ST graft though the same tunnel during the healing period. They considered the tightrope augment was really important factor for their successful surgical procedure and good outcomes. However, in our one-tunnel group, although the sample size was small, we saw no significant difference between patients treated with and without tightrope augmentation. Both of them had a higher re-dislocation rate and achieved the inferior results comparing to the two-tunnel group. From our results, we cannot definitively state that tightrope augmentation is not important and effective for the $\mathrm{CC}$ complex reconstruction, but our results do provide strong evidence that the reconstruction technique (specifically the choice between one or two tunnels) largely impacts the radiographic and clinical outcomes.

The principal limitations of this study are the relative small sample size who met our inclusion criteria and the fact that we did not have preoperative functional scores. Thus, our conclusions are focused on the substantial difference in success rates we saw between the single-tunnel and two-tunnel groups (18\% vs. $70 \%)$, and we have limited ability to assess and compare other aspects of the procedures. In addition, because this was an observational study, our data did not permit an accurate assessment of the time to functional recovery. The two-tunnel technique became a standard technique at our institution at a later date than the single-tunnel technique, and so it is possible that surgeon experience may have played a role in the different outcomes among groups. However, we do not believe this confounding factor would be substantial enough to explain the large difference in the two groups that we observed.

\section{Conclusion}

Anatomical reduction the AC joint and biomechanical reconstruction $\mathrm{CC}$ ligaments are crucial for the optimal joint stability and function. Two-tunnel CC reconstruction with an allogenous ST graft provides superior radiographic and clinical results compared to single-tunnel reconstruction technique.

\section{Abbreviations}

CC ligament: Coracoclavicular ligament; ST tendon: Semitendinosus tendon; AC joint: Acromioclavicular joint; UCLA shoulder rating scale: University of California at Los Angeles shoulder rating scale; ROM: Range of motion.

\section{Competing interests}

The authors declare that they have no competing interests.

\section{Authors' contributions}

ZH and WS designed research; JG, KS and WC analyzed data and performed statistical analysis. All authors read and approved the final manuscript.

\section{Author details}

${ }^{1}$ Department of Orthopaedic Surgery, Third Hospital of Hebei Medical University, Shijiazhuang, Hebei 050051, China. ${ }^{2}$ Department of Orthopaedic Surgery, Geisinger Medical Center, Danville, PA 17822, USA. ${ }^{3}$ Mountain Orthopaedic Trauma Surgeons at Swedish, 701 East Hampden Avenue Suite 515, Englewood, CO 80113, USA.

Received: 27 July 2013 Accepted: 11 August 2014

Published: 15 August 2014

\section{References}

1. Bosworth BM: Acromioclavicular separation: New method of repair. Surg Gynecol Obstet 1941, 73:866-871.

2. Rockwood CA, Williams GR, Young DC: Injuries to the Acromioclavicular Joint. In Fractures in Adults, Volume 2. Fourthth edition. Edited by Rockwood CA, Green DP, Bucholz RW. Philadelphia: Lippincott-Raven Pub Publishers; 1996:1341-1413

3. Horn JS: The traumatic anatomy and treatment of acute acromioclavicular dislocation. J Bone Joint Surg Br 1954, 36:194-1201.

4. Lemos MJ: The evaluation and treatment of the injured acromioclavicular joint in athletes. Am J Sports Med 1998, 26:137-144.

5. Lizaur A, Marco L, Cebrian R: Acute dislocation of the acromioclavicular joint. Traumatic anatomy and the importance of deltoid and trapezius. J Bone Joint Surg Br 1994, 76:602-606.

6. Bannister GC, Wallace WA, Stableforth PG, Hutson MA: The management of acute acromioclavicular dislocation. A randomised prospective controlled trial. J Bone Joint Surg Br 1989, 71:848-1850.

7. Fremerey RW, Lobenhoffer P, Ramacker K, Gerich T, Skutek M, Bosch U: Acute acromioclavicular joint dislocation-operative or conservative therapy? Unfallchirurg 2001, 104:294-299.

8. Kumar S, Sethi A, Jain AK: Surgical treatment of complete acromioclavicular dislocation using the coracoacromial ligament and coracoclavicular fixation: report of a technique in 14 patients. $J$ Orthop Trauma 1995, 9:507-510. 
9. Warren-Smith CD, Ward MW: Operation for acromioclavicular dislocation A review of 29 cases treated by one method. J Bone Joint Surg Br 1987, 69:715-718.

10. Weaver JK, Dunn HK: Treatment of acromioclavicular injuries, especially complete acromioclavicular separation. J Bone Joint Surg Am 1972, 54:1187-1194

11. Jones HP, Lemos MJ, Schepsis AA: Salvage of failed acromioclavic acromioclavicular joint reconstruction using autogenous semitendinosus tendon from the knee: surgical technique and case report. Am J Sports Med 2001, 29:234-237.

12. Nicholas SJ, Lee SJ, Mullaney MJ, Tyler TF, McHugh MP: Clinical outcomes of coracoclavicular ligament reconstructions using tendon grafts. Am J Sports Med 2007, 35:1912-1917.

13. Costic RS, Labriola JE, Rodosky MW, Debski RE: Biomechanical rationale for development of anatomical reconstructions of coracoclavicular ligaments after complete acromioclavicular joint dislocations. Am J Sports Med 2004, 32:1929-1936.

14. Lee SJ, Nicholas SJ, Akizuki KH, McHugh MP, Kremenic IJ, Ben-Avi S: Reconstruction of the coracoclavicular ligaments with tendon grafts: a comparative biomechanical study. Am J Sports Med 2003, 31:648-655.

15. Mazzocca AD, Santangelo SA, Johnson ST, Rios CG, Dumonski ML, Arciero RA: A biomechanical evaluation of an anatomical coracoclavicular ligament reconstruction. Am J Sports Med 2006, 34:236-246.

16. Yoo JC, Ahn JH, Yoon JR, Yang JH: Clinical results of single-tunnel coracoclavicular ligament reconstruction using autogenous semitendinosus tendon. Am J Sports Med 2010, 38:950-957.

17. Guy DK, Wirth MA, Griffin JL, Rockwood CA Jr: Reconstruction of chronic and complete dislocations of the acromioclavicular joint. Clin Orthop Relat Res 1998, 347:138-149.

18. Fu FH, Shen W, Starman JS, Okeke N, Irrgang JJ: Primary anatomic double-bundle anterior cruciate ligament reconstruction: a preliminary 2-year prospective study. Am J Sports Med 2008, 36:1263-1274.

19. Stannard JP, Riley RS, Sheils TM, McGwin G Jr, Volgas DA: Anatomic reconstruction of the posterior cruciate ligament after multiligament knee injuries: a combination of the tibial-inlay and two-femoral-tunnel techniques. Am J Sports Med 2003, 31:196-202.

20. LaPrade RF, Hilger B: Coracoclavicular ligament reconstruction using a semitendinosus graft for failed acromioclavicular separation surgery. Arthroscopy 2005, 21:1277.

21. Tauber M, Gordon K, Koller H, Fox M, Resch H: Semitendinosus tendon graft versus a modified Weaver-Dunn procedure for acromioclavicular joint reconstruction in chronic cases: a prospective comparative study. Am J Sports Med 2009, 37:181-190.

22. Yoo JC, Choi NH, Kim SY, Lim TK: Distal clavicle tunnel widening after coracoclavicular ligament reconstruction with semitendinous tendon: a case report. J Shoulder Elbow Surg 2006, 15:256-259.

23. Salzmann GM, Walz L, Buchmann S, Glabgly P, Venjakob A, Imhoff AB: Arthroscopically assisted 2-bundle anatomical reduction of acute acromioclavicular joint separations. Am J Sports Med 2010, 38(6):1179-1187.

24. Hessmann M, Gotzen L, Gehling H: Acromioclavicular reconstruction augmented with polydioxanonsulphate bands. Surgical technique and results. Am J Sports Med 1995, 23:552-556.

25. Wolf EM, Pennington WT: Arthroscopic reconstruction for acromioclavicular joint dislocation. Arthroscopy 2001, 17:558-563.

26. Mazzocca AD, Spang JT, Rodriguez RR, Rios CG, Shea KP, Romeo AA, Arciero RA: Biomechanical and radiographic analysis of partial coracoclavicular ligament injuries. Am J Sports Med 2008, 36:1397-1402.

27. Hernegger GS, Kadletz R, Tight RV: The revolutionary anatomical Wxation in acromioclavicular joint dislocation VA case report. Tech Shoulder Elbow Surg 2006, 7:86-88.

28. Quereshi F, Hinsche A, Potter D: Arthroscopic "TightRope" stabilization of Neer type 2 clavicle fractures. Injury Extra 2007, 38:133-134.

29. Walz L, Salzmann GM, Fabbro T, Eichhorn S, Imhoff AB: The anatomic reconstruction of $A C$ joint dislocations using two TightRope devices: a biomechanical study. Am J Sports Med 2008, 36:2398-2406.

30. Bargren JH, Erlanger S, Dick HM: Biomechanics and comparison of two operative methods of treatment of complete acromioclavicular separation. Clin Orthop Relat Res 1978, 130:267-272.

31. Fukuda K, Craig EV, An KN, Cofield RH, Chao EY: Biomechanical study of the ligamentous system of the acromioclavicular joint. J Bone Joint Surg Am 1986, 68:434-440.
32. Tienen TG, Oyen JF, Eggen PJ: A modified technique of reconstruction for complete acromioclavicular dislocation: a prospective study. Am J Sports Med 2003, 31:655-659.

33. Scheibel M, Ifesanya A, Pauly S, Haas NP: Arthroscopically assisted coracoclavicular ligament reconstruction for chronic acromioclavicular joint instability. Arch Orthop Trauma Surg 2008, 128:1327-1333.

doi:10.1186/1471-2482-14-53

Cite this article as: Hou et al:: Comparison of single and two-tunnel techniques during open treatment of acromioclavicular joint disruption. BMC Surgery 2014 14:53.

\section{Submit your next manuscript to BioMed Central and take full advantage of:}

- Convenient online submission

- Thorough peer review

- No space constraints or color figure charges

- Immediate publication on acceptance

- Inclusion in PubMed, CAS, Scopus and Google Scholar

- Research which is freely available for redistribution 